\title{
Vortex Organization in a Turbulent Boundary Layer Overlying Sparse Rough- ness Elements
}

MICHELE GUALA, Civil Engineering, University of Minnesota, Minneapolis, MN, USA

Email:mguala@umn.edu

CHRISTOPHER D. TOMKINS, Los Alamos National Laboratory, Los Alamos, NM, USA

Email: ctomkins@lanl.gov

KENNETH T. CHRISTENSEN, Mechanical Science and Engineering, Aerospace Engineering and

Geology, University of Illinois, Urbana, IL, USA

Email:ktc@illinois.edu

RONALD J. ADRIAN, School for Engineering of Matter, Transport and Energy, Arizona State University, Tempe, AZ, USA

Email: rjadrian@asu.edu (Author for correspondence) 


\title{
Vortex Organization in a Turbulent Boundary Layer Overlying Sparse Rough- ness Elements
}

\begin{abstract}
Vortex organization in the outer layer of a turbulent boundary layer overlying sparse, hemispherical roughness elements is explored with two-component particle-image velocimetry (PIV) in multiple streamwise-wall-normal measurement planes downstream and between elements. The presence of sparse roughness elements causes a shortening of the streamwise length scale in the near-wall region. These measurements confirm that vortex packets exist in the outer layer of flow over rough walls, but that their organization is altered, and this is interpreted as the underlying cause of the length-scale reduction. In particular, the elements shed vortices which appear to align in the near-wall region, but are distinct from the packets. Further, it is observed that ejection events triggered in the element wakes are more intense compared to the ejection events in smooth wall. We speculate that this may initiate a self-sustaining mechanism leading to the formation of hairpin packets as a much more effective instability compared to those typical of smooth-wall turbulence.
\end{abstract}

Keywords: Boundary layer turbulence; Wall-bounded shear flow turbulence; Bed roughness; Particle image velocimetry

\section{Introduction}

The rough surfaces encountered in hydraulic flows influence not only the bed friction, but also the structure of the eddies within the flow. Traditionally, the details of the geometry of the roughness elements and the distribution of sizes among the roughness elements are ignored by using the concept on effective sand grain roughness. However, with increasing understanding of the structure of turbulent flow over smooth surfaces (see Adrian and Marusic, 2012, in this issue) there is both the means and the motivation to understand the structure of eddies created by rough walls. The structure is complicated by three processes. First, the flow around and behind single elements can be complex and possibly turbulent in it own right; second, the flow in the free stream above the elements is turbulent, and this certainly has an effect on separation of flow on the elements; third, the wakes of the elements interact more or less strongly depending upon the spacing between the elements. This paper presents a study of a turbulent boundary layer flowing over a sparse periodic array of hemispheres mounted on a smooth surface. Hydrodynamically, such a flow has relevance to various surfaces such as riveted plates or channels with sparsely scattered stones on the bed. The paper focuses on the structure of the turbulent eddies behind and between the hemispheres, and how they change the mean character of structure above the elements.

The impact of surface roughness on the structural paradigm of smooth-wall turbulence is still a topic of intense interest as any alteration of its characteristics may significantly impact how one models momentum and energy transport in such flows. Krogstad and Antonia (1994), for example, computed two-point velocity correlations from cross-wire measurements and found that the inclination angle of $\rho_{u u}$ increased to approximately $38^{\circ}$ for flow over a mesh surface $(\delta / k \approx 50$, $\delta / k_{s} \approx 15, k^{+}=92, k_{s}^{+}=331$, where $k$ is the geometric height of the roughness and $k_{s}$ is the equivalent sand-grain height) compared to the smooth-wall angle of $10-15^{\circ}$ observed in many studies (Brown and Thomas, 1977; Christensen and Adrian, 2001; Christensen and Wu, 2005). In addition, they reported a dramatic decrease in the streamwise extent of velocity and vorticity correlations compared to flow over a smooth wall that persisted well into the outer layer but found little difference in the spanwise extent of these correlations. While weaker streamwise shortening of 
velocity correlations has been observed in other studies of rough-wall turbulence (Raupach et al., 1991; Wu and Christensen, 2007; Volino et al., 2007; Wu and Christensen, 2010), these efforts also reported this shortening to diminish significantly with increasing distance from the wall and eventual consistency with smooth-wall flow outside the roughness sublayer. Such outer-layer similarity is in accordance with Townsend's wall similarity hypothesis which states that surface conditions set the wall shear stress (and hence the friction velocity, $u_{\tau} \equiv \sqrt{\tau_{w} / \rho}$ ) and the boundary-layer thickness, $\delta$, while the turbulence simply adjusts to such conditions in a universal manner. A necessary condition for such similarity to exist in rough-wall turbulence appears to be significant scale separation between the outer length scale of the flow $(\delta)$ and the characteristic height of the roughness (taken as either $k$ or $k_{s}$ ). Jimenez (2004) suggests $\delta / k \gtrsim 50$ for such similarity to exist, while Flack et al. (2005) proposes $\delta / k_{s} \gtrsim 40$. Indeed, many rough-wall turbulence studies exhibit such similarity in the single-point statistics, both for flow over idealized surfaces (Raupach, 1981; Ligrani and Moffat, 1986; Bandyopadhyay and Watson, 1988; Flack et al., 2005; Bakken et al., 2005; Kunkel and Marusic, 2006, among others) as well as for flow over more irregular topographies (Allen et al., 2007; Wu and Christensen, 2007; Mejia-Alvarez and Christensen, 2010b). In contrast, other studies report single-point statistics affected by roughness well into the outer layer (Krogstad et al., 1992; Krogstad and Antonia, 1999; Keirsbulck et al., 2002; Tachie et al., 2000, 2003). However, most of these efforts had weak scale separation between the roughness and the outer length scale of the flow, yielding $\delta / k$ and/or $\delta / k_{s}$ values well below the thresholds proposed by Jimenez (2004) and Flack et al. (2005). ${ }^{1}$

Nakagawa and Hanratty (2001) reported two-point streamwise velocity correlation coefficients computed from ensembles of instantaneous velocity fields acquired by particle image velocimetry (PIV) in channel flow with a wavy bottom wall $(\delta / k \approx 60)$ and found that neither their length scale nor their inclination angle were altered in the presence of the wavy surface. Sabot et al. (1977) studied pipe flow roughened with spanwise fences $(\delta / k=15)$ and found the streamwise integral length scales of the streamwise and wall-normal velocities to change little from smoothwall pipe flow. More recently, Volino et al. (2007) concluded that the outer-layer structure of flow over a fully-rough woven mesh $(\delta / k=71)$ was similar to that observed in smooth-wall flow via comparison of velocity spectra and two-point correlations, though a reduction in streamwise extent of $\rho_{u u}$ by roughness was noted within the roughness sublayer. Similar structural consistencies in the outer layer were also observed in a recent direct numerical simulation (DNS) of channel flow with disturbed walls by Flores et al. (2007). Most recently, Wu and Christensen (2010) conducted PIV measurements of flow over a multi-scale roughness topography replicated from a damaged turbine blade and reported both the instantaneous and the average spatial structure (inferred from two-point velocity correlations) of the outer layer to be qualitatively similar to that of flow over a smooth wall. In contrast, Volino et al. (2009) found the outer-layer structure of a turbulent boundary layer to be more affected by two-dimensional roughness $(\delta / k=32)$. It was concluded that this roughness generates flow structures much larger than $k$ due to the width of the roughness and that the growth of these motions into the outer layer enhanced the turbulent stresses and the integral length scales. These observations highlight the fundamental differences between flow over three-dimensional and two-dimensional roughness as other studies of two-dimensional roughness have similarly reported strong outer-layer modifications in an external flow arrangement (Krogstad and Antonia, 1999; Keirsbulck et al., 2002; Lee and Sung, 2007).

\footnotetext{
${ }^{1}$ In all of the studies of the lengths of structure mentioned here, the lengths are usually measured by the integral length scale of the two-point spatial correlation which is of the order of the flow depth. This scale is not very sensitive to the very large scales of motion (VLSM) (Adrian and Marusic, 2012), because the VLSMs manifest themselves in the long, low-level tails of the correlation, which are often not measured accurately or discarded in the computation of the integral scale.
} 
Although the influence of roughness on the outer layer is still a subject of rather vigorous debate, it is certainly well-accepted that within the roughness sublayer, $\sim 3-5 k$ away from the wall, the turbulence is strongly affected by the surface conditions. Qualitatively, such effects include significant modifications of both the wall shear stress as well as the voracity of the turbulent velocity fluctuations compared to flow over a smooth wall. In particular, the wall-normal velocity fluctuations appear to be most sensitive to rough-wall conditions (Raupach, 1981; Bandyopadhyay and Watson, 1988; Krogstad et al., 1992; Krogstad and Antonia, 1999; Keirsbulck et al., 2002). It has also been reported that these modifications can be highly dependent upon the details of the local surface conditions (Raupach, 1981; Bandyopadhyay and Watson, 1988; Raupach et al., 1991; Krogstad and Antonia, 1999; Keirsbulck et al., 2002; Jimenez, 2004; Mejia-Alvarez and Christensen, 2010a; Mejia-Alvarez et al., 2012) and that such effects may be attributable to the generation of additional intense vortical structures in rough-wall flows via shedding from dominant topographical features (Bandyopadhyay and Watson, 1988).

A subset of the literature on wall roughness documents vortical structures generated by discrete wall-mounted elements (and isolated wall disturbances, like blowing) in both laminar and turbulent wall-bounded flows. Acarlar and Smith (1987a), for example, observed the periodic shedding of hairpin-like vortices from a wall-mounted hemisphere in an otherwise laminar boundary layer for $120<\operatorname{Re}=\hat{u} h / \nu<3400$, where $\hat{u}$ is the streamwise velocity at the tip of the bump and $h$ is the height of the bump. The hairpin structures periodically shed from the hemisphere were observed to convect downstream and this train of vortices generated an elongated low-momentum region in the wake of the element. In addition, a stationary "necklace" vortex was observed to form around the perimeter of the element whose legs extended well downstream of the hemisphere. With regard to the influence of isolated roughness elements in a turbulent boundary layer, Castro and Robins (1977) studied the flow around a rather large surface-mounted cube $(h / \delta=0.1)$ in both laminar and turbulent boundary layers and found that increased turbulence levels upstream of the cube reduced the size of the wake generated downstream of the element. Savory and Toy (1986) also considered the flow downstream of a large surface-mounted element (hemispheres with $h / \delta>0.26$ ) for smoothand rough-wall upstream flows and reported that if vortices are shed from the hemisphere their influence is certainly limited to the region just downstream of the element. They conjectured that the absence of sustained shedding and large-scale wakes was attributable to strong turbulence levels in the vicinity of the element as well as the three-dimensionality of the flow. Similar lack of consistent shedding from and persistent wakes downstream of surface-mounted elements extending into and beyond the log layer in turbulent boundary layers has also been reported in other recent studies (Schofield and Logan, 1990; Martinuzzi and Tropea, 1993; Ryan et al., 2011).

Other studies have considered the influence of much smaller discrete roughness elements in the presence of turbulence, particularly their impact within the near-wall region and the possible generation of sustained wakes as an explanation for the increased turbulence levels observed in the roughness sublayer. Bandyopadhyay and Watson (1988), for example, noted significant differences in the vertical flux of the Reynolds shear stress, $\left\langle u^{\prime} v^{\prime}\right\rangle$, for smooth, 2-D (transversely grooved) rough and 3 -D rough surfaces (Here, $x, y$ and $z$ are the streamwise, wall-normal and spanwise directions, respectively, $u, v$ and $w$ are the velocity components in these respective directions and $(\cdot)^{\prime}$ denotes a fluctuating quantity). In the case of smooth and 2D rough conditions, it was suggested that the net vertical transport of $\left\langle u^{\prime} v^{\prime}\right\rangle$ away from the wall can be accounted for by the existence of hairpin-like vortices that may be shed by the discrete roughness elements in the case of the rough-wall flow. However, the observed transport of $\left\langle u^{\prime} v^{\prime}\right\rangle$ toward the wall in the 3D roughness case was interpreted in terms of an additional vortical structures, a "necklace" vortices, that straddle the 3D roughness elements near their bases. Tomkins (2001) conducted PIV measurements downstream of isolated cylindrical and hemispherical elements immersed in a turbulent boundary layer $(H \approx 0.05-0.15 \delta$; 
$\left.H^{+} \approx 100-300\right)$ and found vortices to be shed downstream of both element types, though a higher density of spanwise vortex signatures consistent with hairpin-like vortices were noted downstream of the hemisphere relative to the cylinder. Finally, George (2005) studied flow around isolated cylindrical elements immersed below the log layer $\left(y^{+}<100\right)$ of a turbulent boundary layer and found that a horseshoe vortex is formed around the element, with the element wake extending tens of diameters downstream and the downwash of high-speed fluid driven by the counter-rotating legs of the horseshoe vortex increasing the local skin friction.

The present effort builds upon the single-element observations of Tomkins (2001) by considering the impact of a sparse array of hemispheres on the structure of smooth-wall turbulence. It also complements the excellent study of dense packed beds of roughness elements and the velocity fields occurring above the bed by Detert et al. (2010). Particle-image velocimetry is used to explore the overall spatial structure of the flow downstream of and between adjacent hemispherical elements. In particular, the fate of the smooth-wall structure developed prior to the rough wall is explored along with possible interactions between this mature structure and that shed locally by the hemispherical elements.

\section{Experiments}

All measurements were made in an Eiffel-type, open circuit, boundary-layer wind tunnel with a documented turbulence intensity of $0.16 \%$ in the free stream (Adrian et al., 2000b). The working section of the tunnel is $6.1-\mathrm{m}$ long, $0.91-\mathrm{m}$ wide and $0.46-\mathrm{m}$ tall within which a 6 - $\mathrm{m}$ long hydraulically smooth flat plate with an elliptically shaped leading edge is suspended $100 \mathrm{~mm}$ above the floor of the tunnel. This boundary-layer surface consists of two separate $3 \mathrm{~m}$-long by $0.91 \mathrm{~m}$-wide flat plates smoothly joined at the streamwise center. Zero-pressure-gradient conditions within $1 \%$ of the free-stream dynamic head were achieved for both surface conditions through fine adjustment of the test-section ceiling height along its length. Fluid properties were assessed from measurements of the atmospheric pressure and air temperature using an ideal gas relation in concert with Sutherland's correlation for kinematic viscosity. The boundary layer was tripped approximately $5 \mathrm{~m}$ upstream of the measurement location with a $4.7 \mathrm{~mm}$-diameter cylindrical rod. The characteristics of this tunnel have been documented in many past studies (Meinhart and Adrian, 1995; Adrian et al., 2000b; Tomkins and Adrian, 2003; Wu and Christensen, 2006, 2007, 2010; Natrajan et al., 2007; Balakumar and Adrian, 2007).

The incoming boundary layer developed over the smooth surface for approximately $45 \delta$ (where $\delta$ is denotes the $99 \%$ boundary-layer thickness at the measurement location for smooth-wall flow) prior to encountering the roughness studied herein. Hemispherical roughness elements were adhered to the existing smooth-wall boundary layer plate in a staggered manner with spacings of $\delta$ and $\delta / 2$ in the streamwise and spanwise directions, respectively as illustrated in figure 1. Two different hemisphere radii were investigated: $7.2 \mathrm{~mm}$ radius (denoted case ' $\mathrm{A}$ ') and $3.6 \mathrm{~mm}$ radius (denoted case 'B'). Based on the sparse spacing of the elements in both the 'A' and 'B' cases, both roughness types can be categorized as k-type roughness as quantified by the roughness density, $\lambda \equiv S_{f} / S<$ 0.15 , where $S_{f}$ is the projected frontal area and $S$ is the total surface area. The blockage ratio, $\delta / k$, was 11.8 and 23.6 for cases A and B, respectively. Table 1 summarizes many of the parameters associated with the two rough-wall flows along with the characteristics of a baseline smooth-wall experiment performed as well, including the downward shift of the mean velocity profile in inner scaling termed the roughness function, $\Delta U^{+}$.

Particle image velocimetry (PIV) measurements were performed in various streamwise-wallnormal $(x-y)$ planes as illustrated in figure 1 to document the impact of the roughness on the 


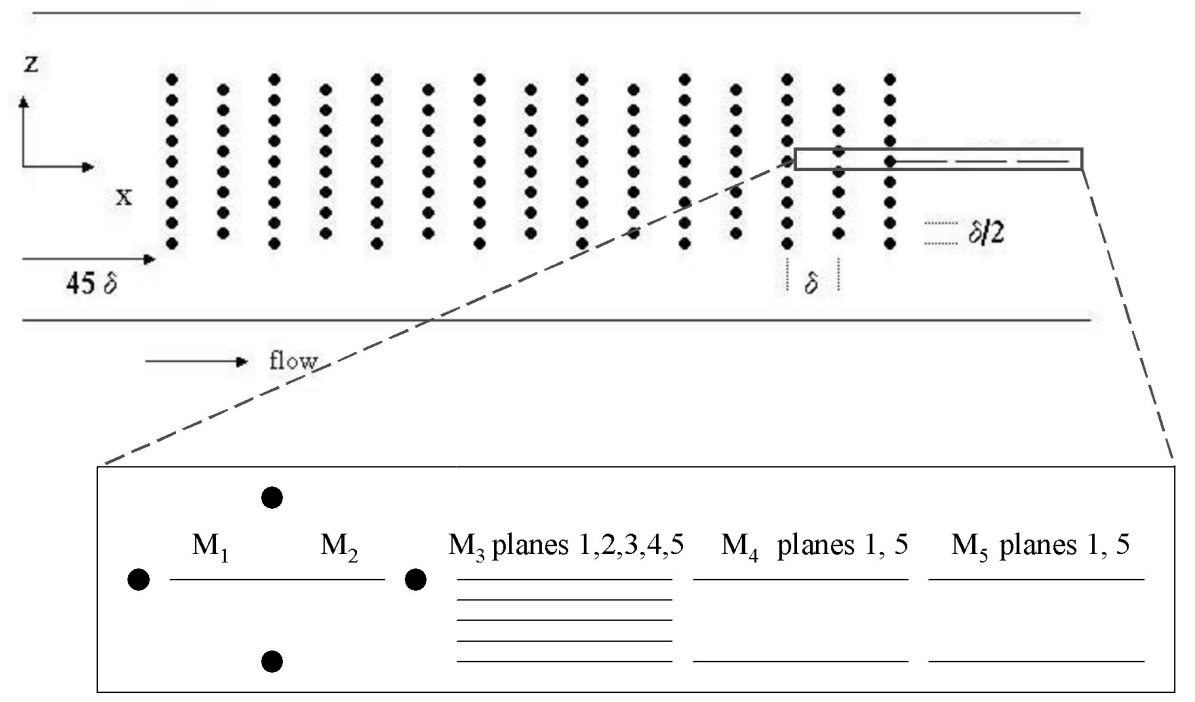

Figure 1: Schematic illustrating top view of staggered arrangement of hemispherical roughness elements on the smooth boundary-layer plate. Flow is from from left to right and measurement locations are indicated as $M_{i}$ in the streamwise direction and as planes $j$ in the spanwise direction.

\begin{tabular}{cccccccccc}
\hline & $\delta[\mathrm{mm}]$ & $u_{\tau}\left[\mathrm{ms}^{-1}\right]$ & $U_{\infty}\left[\mathrm{ms}^{-1}\right]$ & $k[\mathrm{~mm}]$ & $k^{+}$ & $\lambda$ & $k_{s}^{+}$ & $\delta / k_{s}$ & $\Delta U^{+}$ \\
\hline smooth & 82 & 0.40 & 11.4 & & & & & & \\
hem B & 85 & 0.44 & 11.4 & 3.6 & 100 & 0.01 & 20 & 124 & 3 \\
hem A & 86 & 0.57 & 11.4 & 7.2 & 200 & 0.04 & 200 & 15 & 9 \\
\hline
\end{tabular}

Table 1: Relevant experimental parameters.

flow. A dual-cavity, pulsed Nd:YAG laser (New Wave Gemini) with an energy of $120 \mathrm{~mJ} / \mathrm{pulse}$ and a pulse duration of $5 \mathrm{~ns}$ was used to form the $0.5 \mathrm{~mm}$ thick laser light sheets that were introduced into the wind tunnel through the transparent boundary-layer plate from below that illuminated the $1 \mu \mathrm{m}$ olive oil droplets utilized as tracer particles in the $x-y$ planes of interest. The scattered light from the particles was imaged with a $1 \mathrm{k} \times 1 \mathrm{k}$ pixel, 8-bit frame-straddle CCD camera (TSI PIV-CAM 10-30) focused on the light sheet with a 0.077 magnification and viewed the lightsheet in side scatter through the transparent side wall of the wind tunnel over a field of view of $l_{x} \times l_{y}=1.18 \delta \times 1.03 \delta$.

The PIV images were interrogated using a cross-correlation method implemented in the PIV Sleuth software (Christensen et al., 2000). Over 500 image pairs were acquired at each measurement location. The relatively large field of view imaged onto the rather sparse pixel array meant most particle-image diameters did not exceed 1 pixel when the camera was perfectly focused. To avoid significant issues associated with the peak locking effect (Christensen, 2004), the images were acquired in a slightly defocused arrangement to broaden the particle-image diameter to nearly 2 pixels. To limit noise associated with this defocussing, the PIV images were low-pass filtered with a narrow Gaussian filter $(3 \times 3$ mask and a kernel of 1.0 pixels). These image pairs were then interrogated using a $20 \times 16$ pixel first interrogation window and a larger $28 \times 24$ pixel second interrogation window offset in the streamwise direction by 8 pixels to minimize bias errors associated with loss of particle-image pairs. The first interrogation windows were overlapped by $50 \%$. Details of the PIV interrogation parameters are reported in table 2 . The resulting velocity vector fields were then validated using standard deviation and magnitude difference comparisons to remove erroneous 


\begin{tabular}{cccccccc}
$\Delta x^{+}$ & $\Delta y^{+}$ & $l_{x} / \delta$ & $l_{y} / \delta$ & $1^{\text {st }}$ win & $2^{\text {nd }}$ win & offset & $\Delta t$ \\
62 & 50 & 1.18 & 1.03 & $20 \times 16$ & $28 \times 24$ & 8 pix & $100 \mu s$ \\
\hline
\end{tabular}

Table 2: PIV interrogation parameters.

velocity vectors. Erroneous vectors were replaced either with alternate choices determined during interrogation or were interpolated at locations with at least $50 \%$ of neighbors present. Finally, each velocity field was low-pass filtered with a narrow Gaussian filter to reduce noise from random PIV interrogation errors.

\section{Results}

\subsection{Reduction of streamwise length scales}

Two-point correlations of streamwise velocity are utilized to explore the impact of the discrete hemispherical elements on the average spatial structure of the flow. These correlations were computed in each of the measurement planes illustrated in figure 1 using the fluctuating streamwise velocity defined as the total velocity minus the mean velocity profile in each respective plane. The two-point correlations were then computed as

$$
\rho_{u u}\left(r_{x}, y ; y_{\mathrm{ref}}\right)=\frac{\left\langle u^{\prime}\left(x, y_{\mathrm{ref}}\right) u^{\prime}\left(x+r_{x}, y\right)\right\rangle}{\sigma_{u}\left(y_{\mathrm{ref}}\right) \sigma_{u}(y)},
$$

where $r_{x}$ is the spatial separation in the streamwise direction, $\langle\cdot\rangle$ denotes averaging across the ensemble as well as in the streamwise direction and $\sigma_{u}$ is the root-mean-square of the streamwise velocity as a function of wall-normal position.

Figure 2(a) presents $\rho_{u u}$ at $y_{\text {ref }}^{+}=214$ for flow on a smooth wall while figures 2(b)-(d) illustrate $\rho_{u u}$ for flow at downstream location M3 directly behind, a quarter spacing offset in the spanwise direction and midway between two A elements, respectively. Note that this wall-normal reference position is comparable to the inner-scaled height of the A hemispheres. The smooth-wall results display the typical streamwise-elongated coherence and slight inclination away from the wall for $\rho_{u u}$ as reported in previous studies. These average spatial characteristics are consistent with those of instantaneous hairpin vortex packets often observed in the outer-layer of smooth-wall turbulence (Adrian et al., 2000b). A substantial reduction in the streamwise coherence of $\rho_{u u}$ is noted in the measurements immediately downstream of the A hemisphere [figure 2(b)]. However, the long streamwise extent of the weaker correlation levels (0.2, for example) remains intact, indicating that this correlation is likely a superposition of existing large-scale motions away from the wall and smaller-scale structures shed from the upstream hemisphere. Furthermore, measurements at quarter and half spanwise spacings show much less influence of the roughness wherein the correlation contours appear quite similar to those of smooth-wall flow, save for a slight broadening of the wall-normal coherence. The diminished coherence quickly reverts back toward the smooth-wall coherence with increasing spanwise distance form the element. In the case of the B hemispheres (half the height of the A hemispheres), figure 3 illustrates a much weaker impact of these elements on the flow immediately downstream of the hemispheres [figure 3(b)] where the streamwise coherence is only slightly reduced (see 0.4 contour level compared to smooth-wall flow, for example) and the results at quarter and half spanwise spacings [figures 3(c) and 3(d)] are nearly indistinguishable 

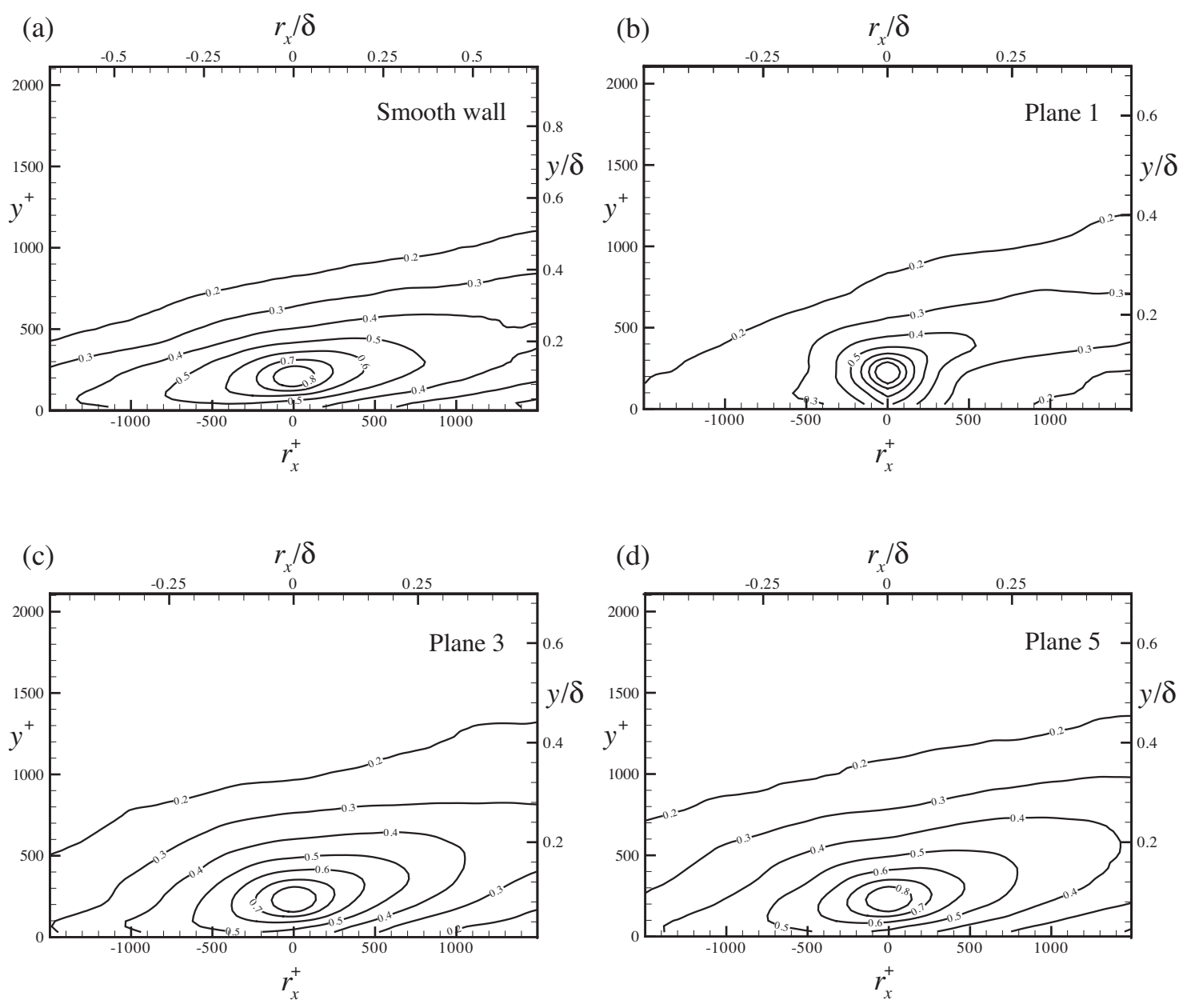

Figure 2: $\rho_{u u}\left(r_{x}^{+}, y^{+} ; y_{\text {ref }}^{+}\right)$for hemisphere A at $y_{\text {ref }}^{+}=214$ at streamwise location $M_{3}$ at different spanwise locations. Plane 1 (b) is located downstream the element, plane 5 (d) is located midway between two elements, and plane 3 (c) is at an intermediate location; smooth wall results for comparison are provided in (a).

from the smooth-wall results [figure 3(a)] (But, note that the B hemispheres are one-half the height of $y_{\text {ref }}$ in these figures.).

Modifications of the streamwise coherence reflected in $\rho_{u u}$ can be quantified by plotting $L_{x}=$ $\left.2 r_{x}\right|_{\rho_{u u}=0.5}$ as a function of $z / D_{z}$ (spanwise measurement plane position) and $y_{\text {ref }}^{+}$. With wall-normal position (figure 4 ), the A hemispheres clearly induce a more dramatic reduction in $L_{x}$ compared to smooth-wall flow, most notably close to the wall for the measurement planes spanwise-aligned behind elements (Planes 1 and 2). With increasing distance from the wall $L_{x}$ becomes closer to the smooth-wall results, though a notable decrease in streamwise coherence is still observed in the five measurement planes downstream of the A elements at $y_{\text {ref }}^{+}=400$. Thus, even the spatial structure of the outer layer is affected by the roughness elements. The wall-normal trends for the $\mathrm{B}$ hemispheres are similar to those of the A hemispheres, though the reduction in streamwise coherence is much less pronounced. Nevertheless, there still exists a reduction in the spatial coherence well into the outer layer of the flow. The trends in $L_{x}$ with spanwise position at fixed $y$ (figure 5) verifies the strongest impact of the elements is felt along the mid plane of the elements in both the A and B hemispheres. 

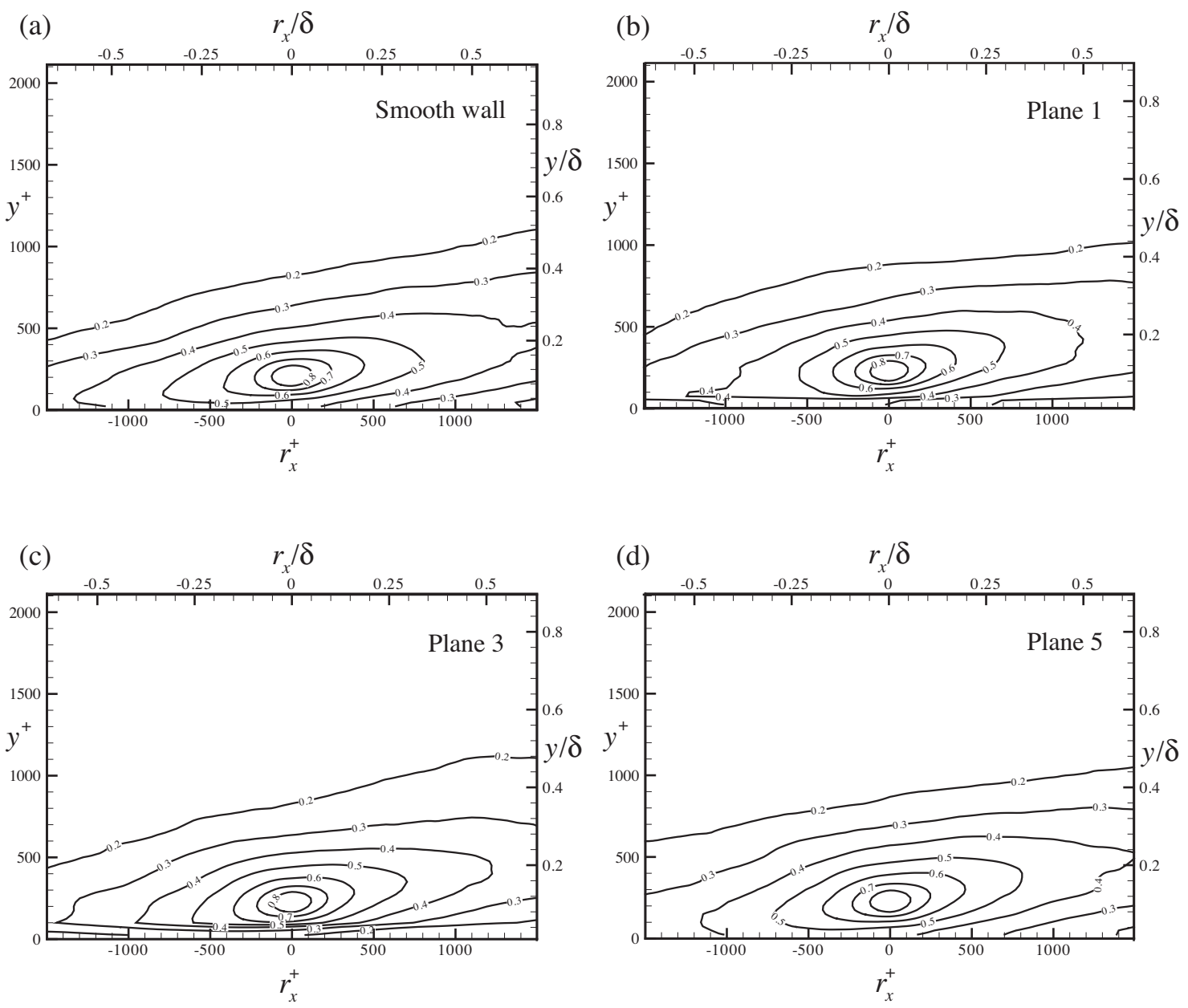

Figure 3: As in figure 2 but for hemisphere B.
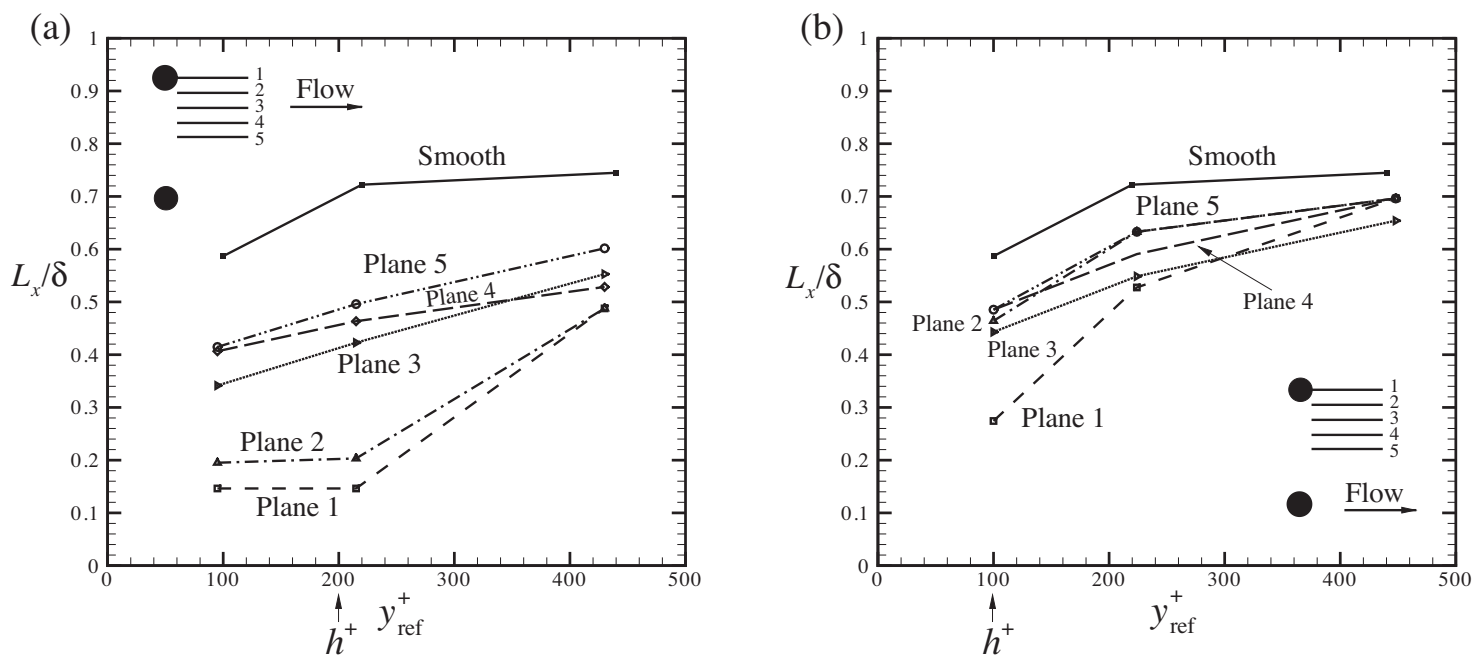

Figure 4: Streamwise length scales corresponding to $\rho_{u u}\left(r_{x}^{+}, y^{+}=y_{\text {ref }}^{+}\right)=0.5$ plotted as a function of $y^{+}$for hemisphere A (a) and B (b) at streamwise location $M_{3}$ and different spanwise locations. 
(a)

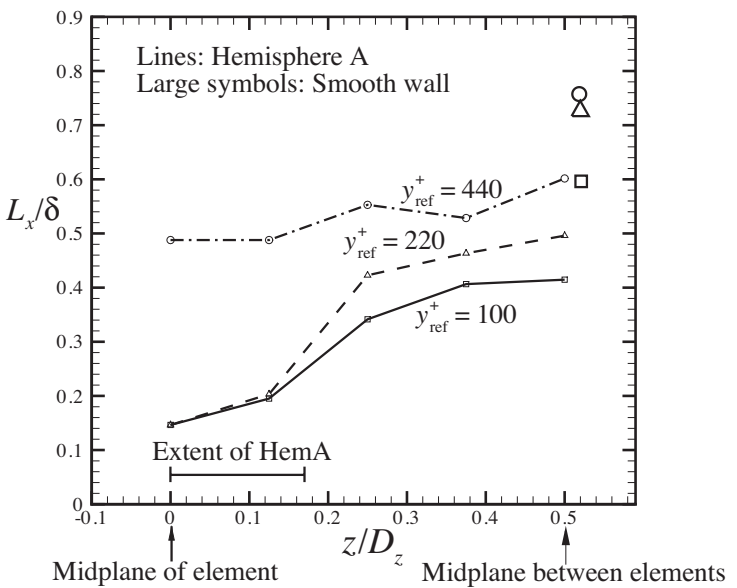

(b)

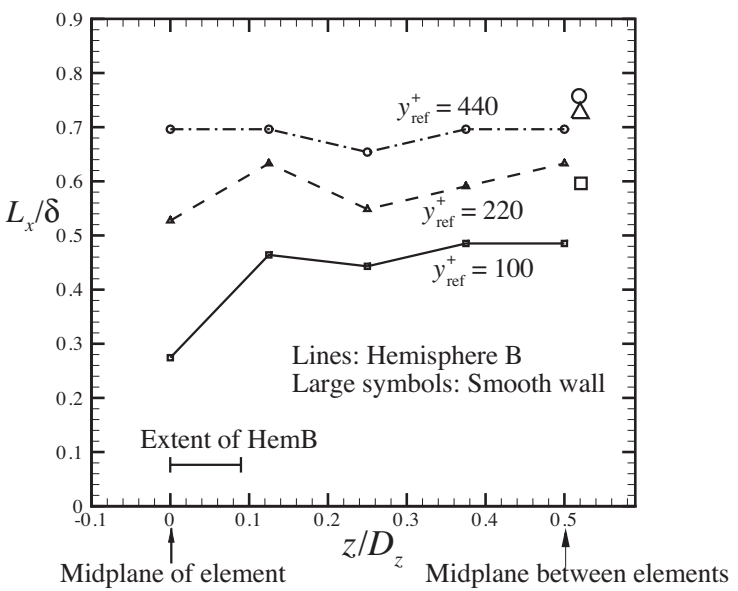

Figure 5: Streamwise length scales corresponding to $\rho_{u u}\left(r_{x}^{+}, y^{+}=y_{\text {ref }}^{+}\right)=0.5$ plotted as a function of $z / D_{z}$ for hemisphere A (a) and B (b) at streamwise location $M_{3}$.

Taken together, the two-point correlation results indicate that ramp-like, hairpin packet structures still populate the flow despite the occurrence of periodically arranged hemispherical elements at the wall based on the elongated streamwise coherence of $\rho_{u u}$ everywhere except immediately downstream of the roughness elements. Along the mid plane of the roughness elements, it is also evident that smaller-scale structures are shed from the hemispheres and the impact of these vortical structures is to reduce the streamwise coherence, most notably behind the element.

\subsection{Vortical activity downstream of elements and in the outer layer}

Figure 6 presents an instantaneous velocity field downstream of a B element in plane 1 (aligned with the spanwise center of the element) with a constant advection velocity of $U_{c}=0.72 U_{\infty}$ removed to reveal vortical structures. Contours of swirling strength $\lambda_{c i}$ are shown in the background to highlight the locations of spanwise vortex cores. Swirling strength is the imaginary part of the complex eigenvalues of the local velocity gradient tensor and is a marker of local rotation (Zhou et al., 1999). A two-dimensional version of $\lambda_{c i}$ is computed in the measurement plane from the in-plane velocity gradients (Adrian et al., 2000a). This field illustrates the simultaneous occurrence of a ramp-like pattern consistent with outer-layer vortex organization above vortices that appear to have been shed from element B. Similar patterns are noted in many of the instantaneous velocity fields acquired for both the A and B elements, indicating a coexistence of outer-layer structure reminiscent of smooth-wall flow with vortices shed locally from the roughness elements.

Figure 7(a) presents an instantaneous velocity field directly downstream of element A with a constant advection velocity $U_{c}=0.72 U_{\infty}$ removed. A train of spanwise vortices is evident downstream of the A element with little inclination away from the wall. The vortices are shed by the hemisphere and evolve downstream parallel to the wall. The streamwise spacing of these vortices is not regular, indicating that this shedding is highly unsteady. This shedding behavior is further confirmed in figure 7(b) which presents contours of the ensemble-averaged $\lambda_{c i},\left\langle\lambda_{c i}\right\rangle$, field downstream of the A element. There exists an intense region of $\left\langle\lambda_{c i}\right\rangle$ emanating from the hemisphere and extending several diameters downstream.

The statistical signatures of the structural patterns noted in figures 6 and 7 are explored through calculation of two-point correlation coefficients of $\lambda_{c i}, \rho_{\lambda \lambda}\left(r_{x}, y ; y_{\text {ref }}\right)$, as presented in figures 8 and 


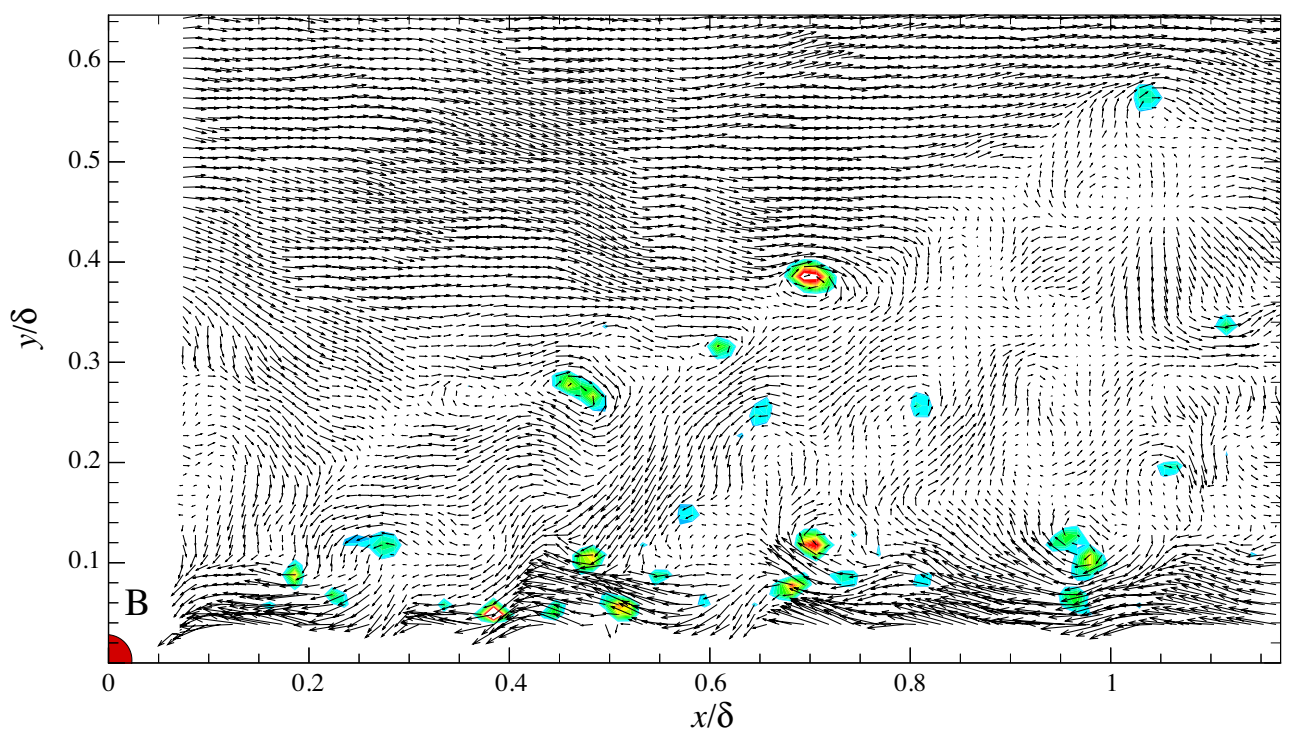

Figure 6: Instantaneous flow field downstream of hemisphere B at location M1. The subtracted convection velocity is $U_{c}=0.72 U_{\infty}$. Iso-contours of $\lambda_{c i}$ are superimposed on the velocity vector field. We note two major vortical patterns: one parallel to the wall located at about $y / \delta=0.1$ and one inclined extending to $y / \delta=0.5$.

9 for the $\mathrm{A}$ and $\mathrm{B}$ elements, respectively, at varying wall-normal and spanwise positions. The smooth-wall correlations of $\lambda_{c i}$ highlight the expected streamwise alignment of spanwise vortices within hairpin packets with spatially compact regions of high correlations both above and downstream as well as below and upstream of the primary correlation peak forming a ramp-like pattern at an inclination of $12-14^{\circ}$ away from the wall. This pattern is interpreted as the statistical signature of the coherent alignment of hairpin vortices into larger-scale packets. In contrast, $\rho_{\lambda \lambda}$ computed in plane 1 (directly downstream of the elements) shows little indication of ramp-like structures. Rather, these correlations reflect small-scale coherence likely associated with the individual vortices shed from the hemispheres. This effect is more pronounced in the case of the A elements which are twice as large as the B elements and therefore shed relatively larger-scale vortices which have a broader region of wall-normal influence. Notably, these correlations directly downstream of the elements do not reflect substantial correlation between consecutively-shed vortices as such correlation would manifest as a region of elongated coherence streamwise coherence in $\rho_{\lambda \lambda}$. In planes 3 and 5 (in-between spanwise-adjacent roughness elements), however, the signature of ramp-like structures is more apparent, albeit slightly less pronounced than in smooth-wall flow. Nevertheless, the structures shed from hemispheres upstream of these measurement locations do not overwhelm larger-scale vortex organization.

\subsection{Statistical signature of hairpin packets overlying the rough wall}

One way to explore the persistence of re-occurring turbulent structures in the flow field is to estimate the average fluctuating velocity field conditioned on the occurrence of such structures. Following Christensen and Adrian (2001), we chose to condition this average on the occurrence of a non-zero value of $\lambda_{c i}$ at a given location $\mathbf{x}$, representing the occurrence of a spanwise vortex at this location. 

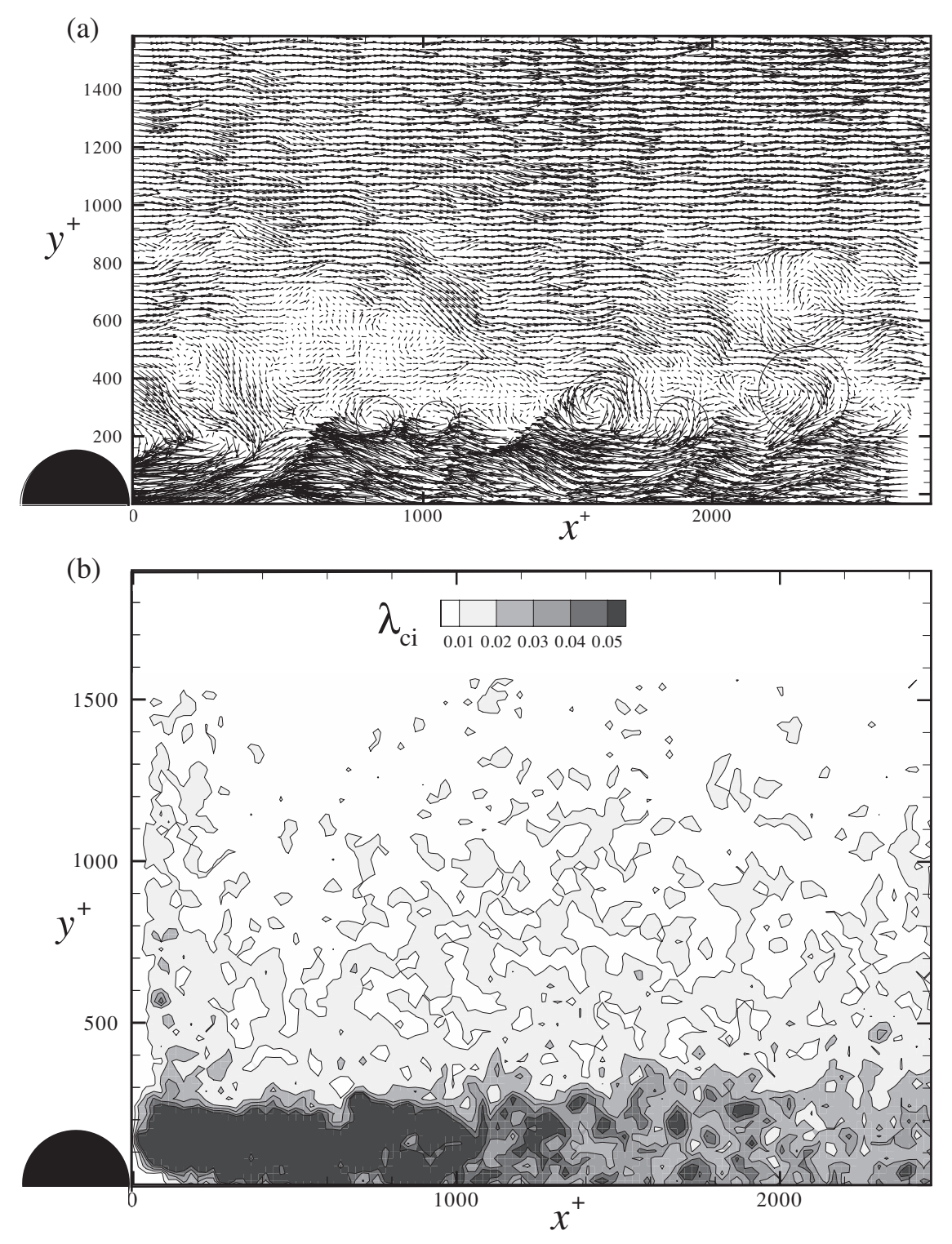

Figure 7: (a) Instantaneous velocity field downstream of an A element at streamwise location $M_{3}$ and in plane 1. A convection velocity of $U_{c}=0.72 U_{\infty}$ is removed. (b) Ensemble-averaged $\lambda_{c i}$ field in plane from (a).

Stochastic estimation (Adrian and Moin (1988)) provides the best linear estimate, in a mean-square sense, of the conditional average of the vector velocity vector at a point $t e x t b f x^{\prime}$ given the swirling strength at textbf $x$ :

$$
\left\langle u_{j}^{\prime}\left(\mathbf{x}^{\prime}\right) \mid \lambda_{c i}(\mathbf{x})\right\rangle \approx L_{j} \lambda_{c i}(\mathbf{x})
$$

. In this equation $u_{j}^{\prime}$ represents the fluctuating velocity components at $\mathbf{x}^{\prime}$ ( $j=1,2,3$ are streamwise, wall-normal and spanwise, respectively). The kernel $L_{j}$ is determined by minimizing the meansquare error between the estimate and the conditional average, leading to the following relationship:

$$
\left\langle u_{j}^{\prime}\left(\mathbf{x}^{\prime}\right) \mid \lambda_{c i}(\mathbf{x})\right\rangle \approx \frac{\left\langle u_{j}^{\prime}\left(\mathbf{x}^{\prime}\right) \lambda_{c i}(\mathbf{x})\right\rangle}{\left\langle\lambda_{c i}(\mathbf{x}) \lambda_{c i}(\mathbf{x})\right\rangle} \lambda_{c i}(\mathbf{x})
$$


(a)

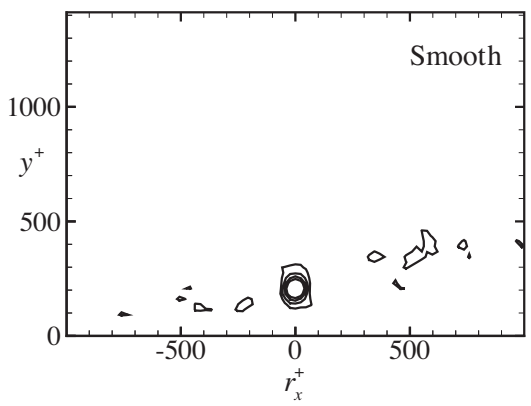

(c)

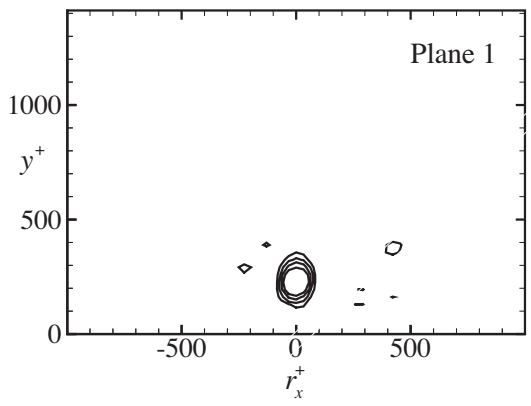

(e)

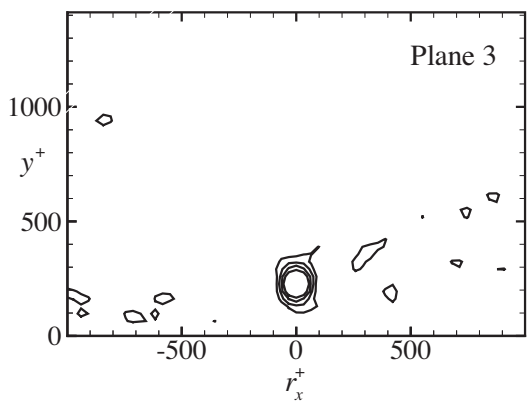

(g)

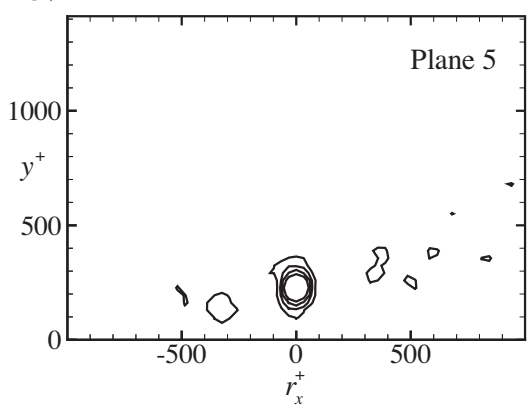

(b)

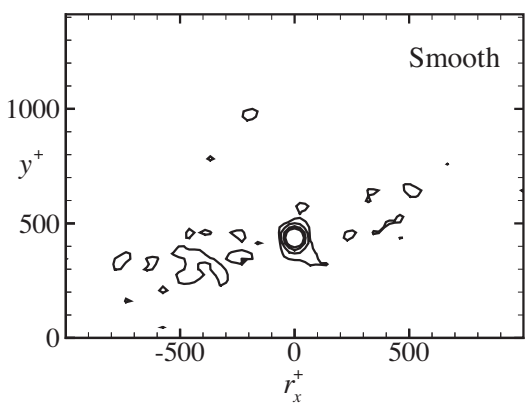

(d)

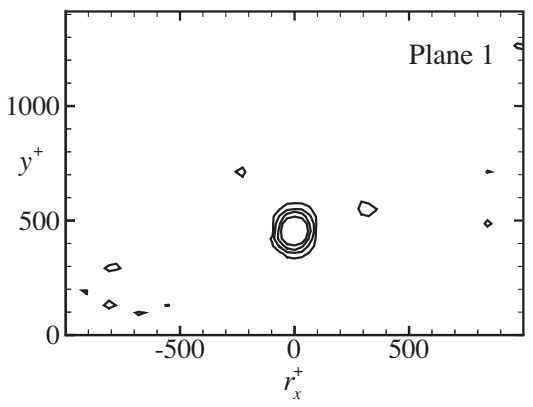

(f)

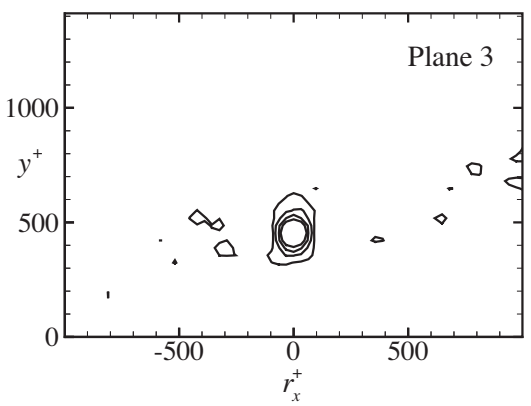

(h)

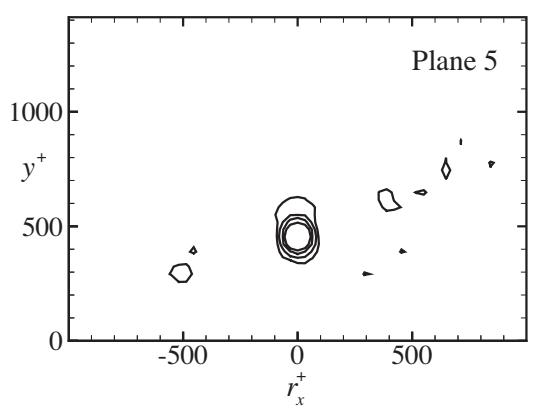

Figure 8: $\rho_{\lambda \lambda}\left(r_{x}^{+}, y^{+} ; y_{\text {ref }}^{+}\right)$at streamwise location $M_{3}$ and different spanwise locations at $y_{\text {ref }}^{+}=225$ (left) and $y_{\text {ref }}^{+}=450$ (right) for hemisphere A.

where the non normalized two-point correlation between swirling strength and velocity can be written as

$$
\left\langle u_{j}^{\prime}\left(\mathbf{x}^{\prime}\right) \lambda_{c i}(\mathbf{x})\right\rangle=\left\langle\lambda_{c i}\left(x, y_{\mathrm{ref}}\right) u_{j}^{\prime}\left(x+r_{x}, y\right)\right\rangle
$$


(a)

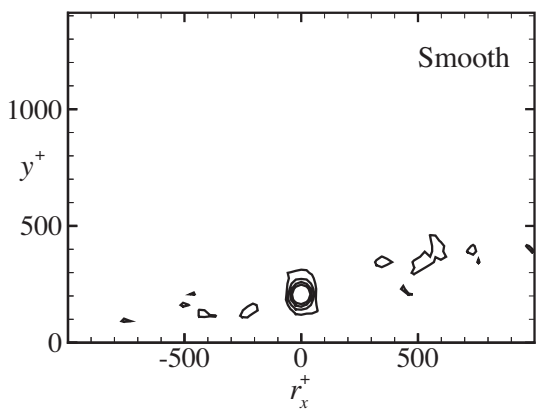

(c)

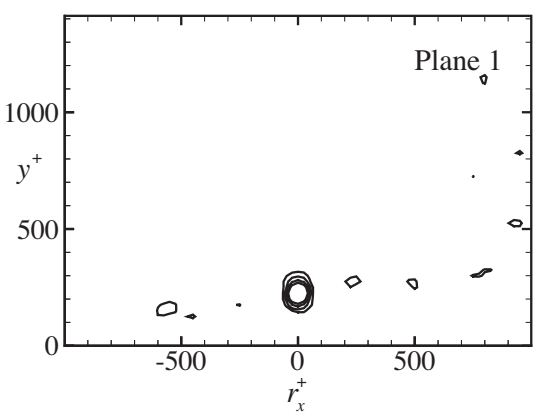

(e)

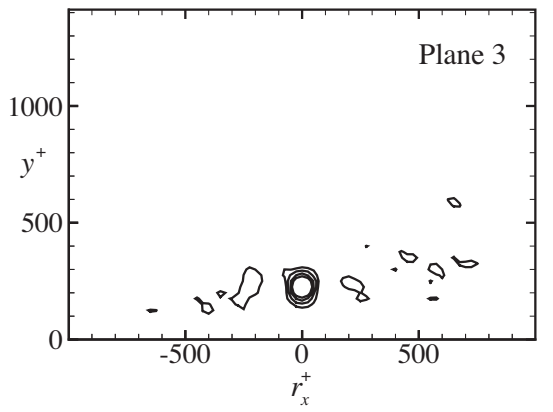

(g)

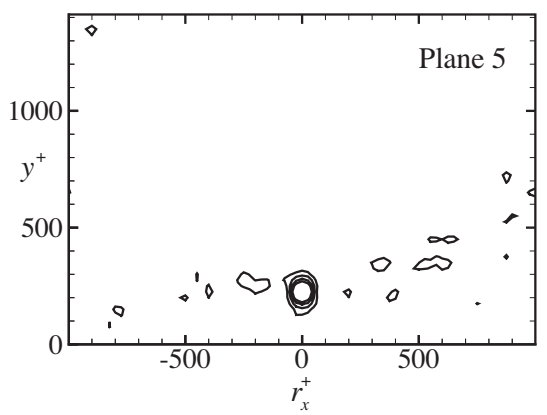

(b)

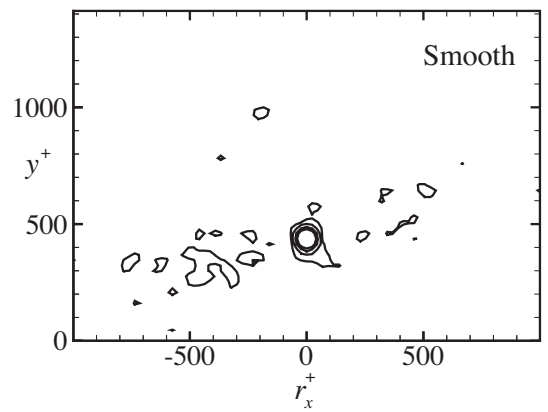

(d)

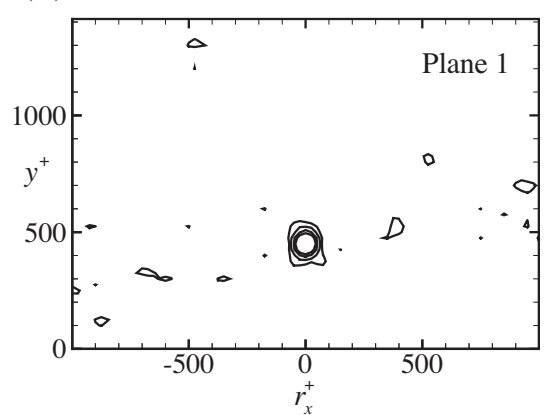

(f)

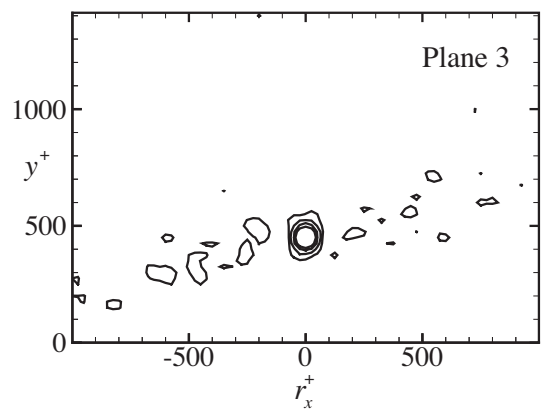

(h)

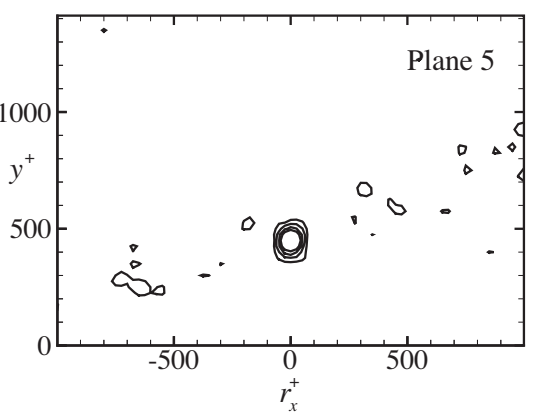

Figure 9: $\rho_{\lambda \lambda}\left(r_{x}^{+}, y^{+} ; y_{\text {ref }}^{+}\right)$at streamwise location $M_{3}$ and different spanwise locations at $y_{\text {ref }}^{+}=225$ (left) and $y_{\text {ref }}^{+}=450$ (right) for hemisphere B.

Since the event is unambiguously determined by the scalar variable $\lambda_{c i}(\mathbf{x})$ one can assume any nonzero value for such a scalar event. The estimate of the conditionally-averaged velocity field is then determined by a two-dimensional vector field, whose magnitude is globally amplified or attenuated by the choice of $\lambda_{c i}(\mathbf{x})$. The resulting $2 \mathrm{D}$ pattern is therefore affected by the choice of a different 
swirling strength intensity only through a scaling factor. Furthermore, the stochastically-estimated velocity field is strongest around the event point and weaker in its neighborhood. For the above reasons, in order to visualize the conditioned velocity field even far away from the event location, the length of each vector is normalized by its magnitude, yielding a direction field. This procedure allows one to obtain information only about the persistent pattern of turbulent structures with no distinction as to their magnitude. We show the LSE of the above conditional average, assuming streamwise homogeneity, in figure 10. These results reveal inclined shear-layer-like structures for event locations far from the rough wall, reminiscent of the inclined interface characteristic of hairpin vortex packets in instantaneous velocity realizations. These patterns are quite consistent with the results of Christensen and Adrian (2001) for smooth-wall flow, though the inclination angle is larger in the present rough-wall flow. Nevertheless, the persistence of this characteristic outer-layer vortex organization supports the existence of hairpin packets in this rough-wall flow. As one moves closer to the wall, this pattern is lost, as the flow is likely heavily influenced by the structures shed locally in the element wakes.

\subsection{Distinguishing roughness-induced structure from outer-layer structure}

From the two previous sections it can be concluded that i) hairpin packets typical of smooth-wall turbulence survive over the sparse distribution of roughness elements and ii) that each element sheds structures that scale with the elements height and evolve parallel to the wall for at least $1 \delta$. We also observed that the alteration of hairpin packets may manifest itself in the alteration of the streamwise alignment of individual vortices. This may be due to two, possibly interacting, processes: alteration in the spanwise direction, possibly related to the spanwise modulation of the wake regions of each element and alteration in the wall-normal direction due to the steepening of the inclination angle of the ramp-like structure characteristic of the packets. The steepening is similar to flow visualization of the bursts from a gravel bed (Roy et al., 2004, Fig. 16) which showed structures having growth angles similar to the $\sim 15^{\circ}$ angle of hairpin vortex packets, followed by steeper structures inclined more than $45^{\circ}$. The steepening could be due to two different reasons, again related to the different length scales of the flow. The presence of the hemisphere as a solid obstacle extending into the logarithmic layer may deflect the packets upwards into the outer layer. Moreover, the self-sustaining mechanism leading to the formation of the packet could be also altered due to the frequent occurrence of tightly spaced $Q_{2}$ events near the wall due to vortices shed from the roughness elements. This is indeed related to the propagation of the scale $k_{s}$ in the whole outer layer. This picture is in general agreement with the suggestion by Grass (1971) that intense ejection events could be responsible for the reduction of the streamwise integral length scale. In order to investigate statistically the evolution of vortex structures from the wall towards the outer layer in the region downstream of the elements we need to relax the assumption of spatial homogeneity in the streamwise direction and study two-point correlation functions and LSE, as a function of both

$x_{\text {ref }}$ and $y_{\text {ref }}$. We show in figure 11, that $\rho_{u u}$ is strongly dependent upon $x_{\text {ref }}$ and $y_{\text {ref }}$, as it recovers its typical inclined shape as one moves further downstream of the element wakes. Closer to the elements, the spatial coherence is drastically reduced, indicating a strong influence of smaller-scale structures likely shed from the elements. Thus, it is clear that in the element wake the assumption of streamwise homogeneity is not fully justified.

Two-point inhomogeneous correlations of $\lambda_{c i}$ at various $x_{\text {ref }}$ and $y_{\text {ref }}$ (figure 12) demonstrate clearly that two different populations of organized structures exist: a series of vortices spaced $200-300 y_{*}$ (where $y_{*}$ is the viscous length scale) evolving parallel to the wall and inclined ramplike patterns in the outer layer. We interpret these inclined outer-layer structures as hairpin packets typical of smooth-wall turbulence, though altered. It should be noted that if the latter were tied 


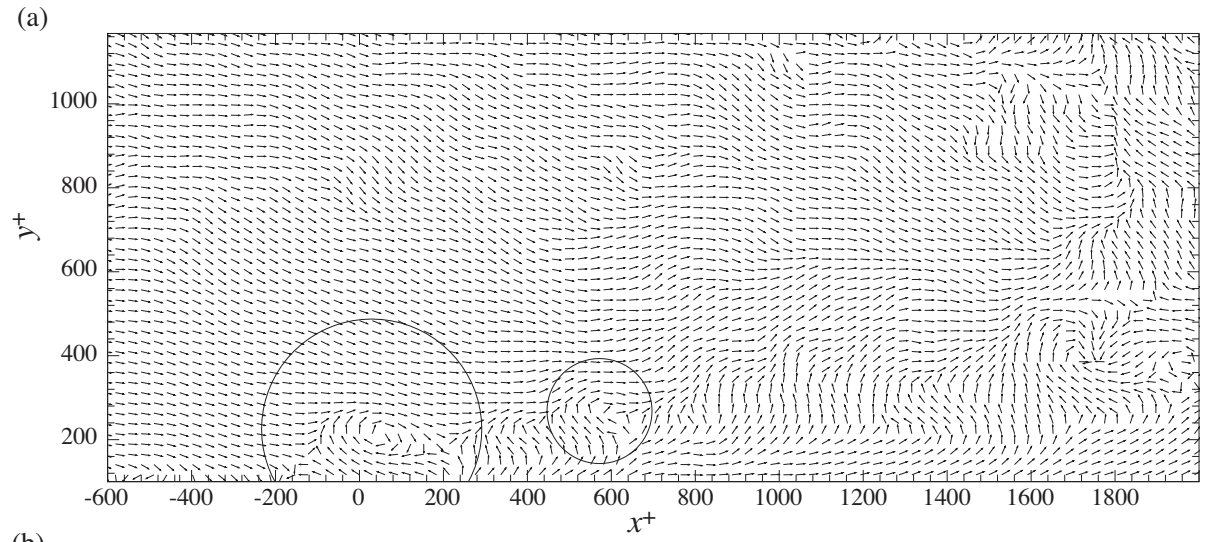

(b)
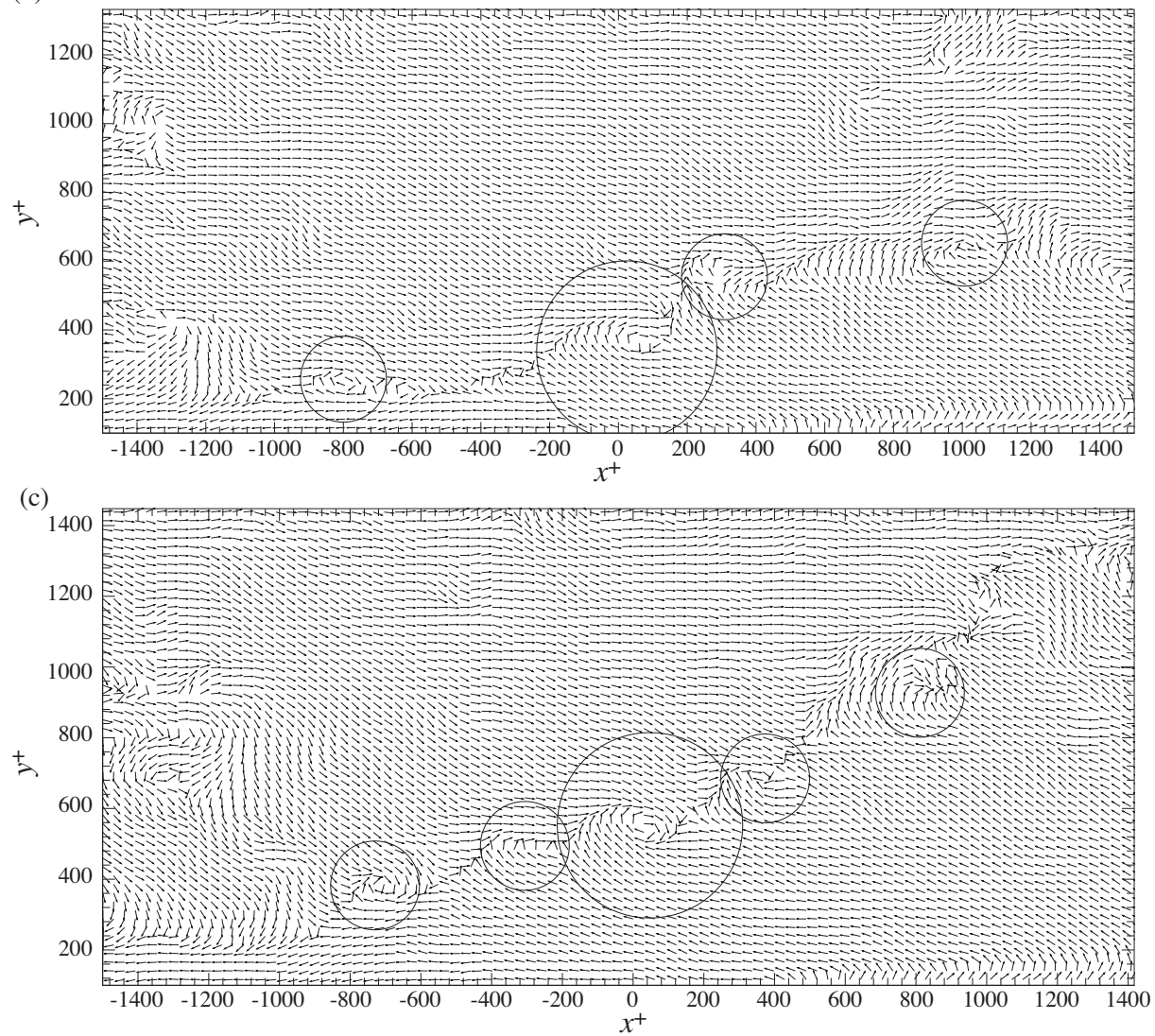

Figure 10: Linear stochastic estimate of the conditionally-averaged flow field given a spanwise vortex at streamwise location $M_{1}$ and (a) $y_{\text {ref }}^{+}=214$, (b) $y_{\text {ref }}^{+}=361$ and (c) $y_{\text {ref }}^{+}=532$ for hemisphere B. The event is indicated with the large circle in each field.

spatially to the elements, e.g. if they were shed from the elements upstream of the measurement location, they would not persist in the homogeneous LSE as shown in figure 10.

Finally, we studied the effect of hemisphere $\mathrm{A}$ on the $Q_{2}$ events in the near-wall region $\left(y^{+}=\right.$ 100). We show in figure 13, that statistically, as we move towards the wake of the element, the intensity of the $Q_{2}$ events becomes much more significant compared to that of smooth-wall flow. We can speculate that an enhanced $Q_{2}$ event may trigger more rapidly the formation of hairpins both downstream and upstream of the original disturbance, which, in this case, is a hairpin-like 
(a)

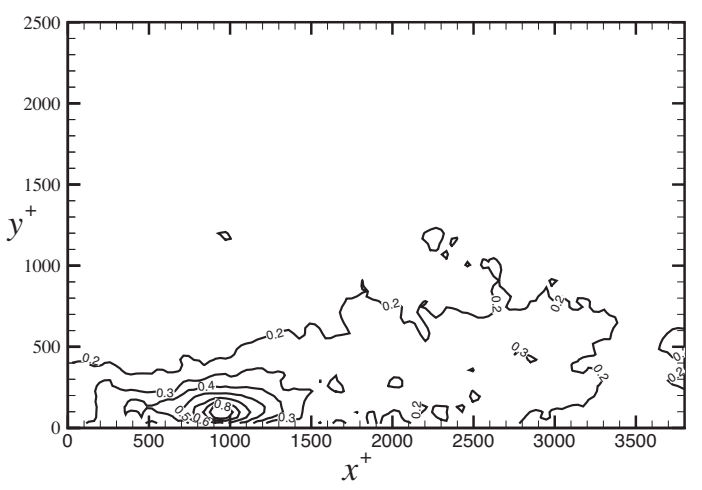

(c)

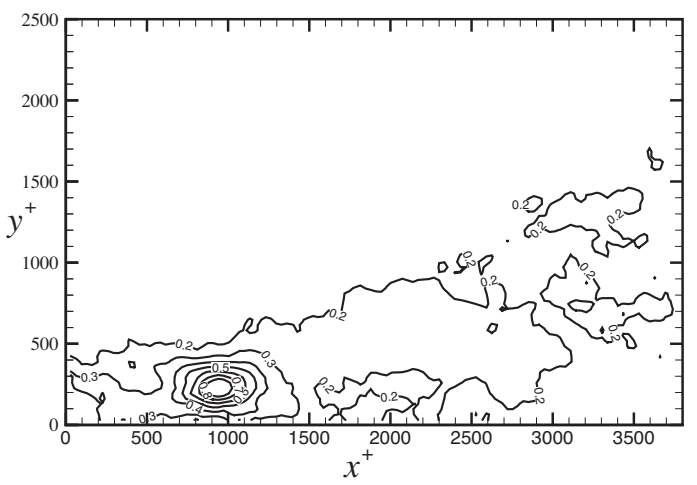

(b)

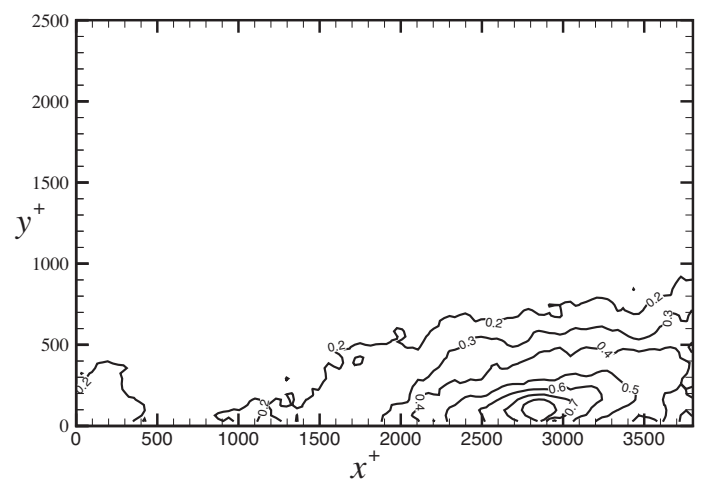

(d)

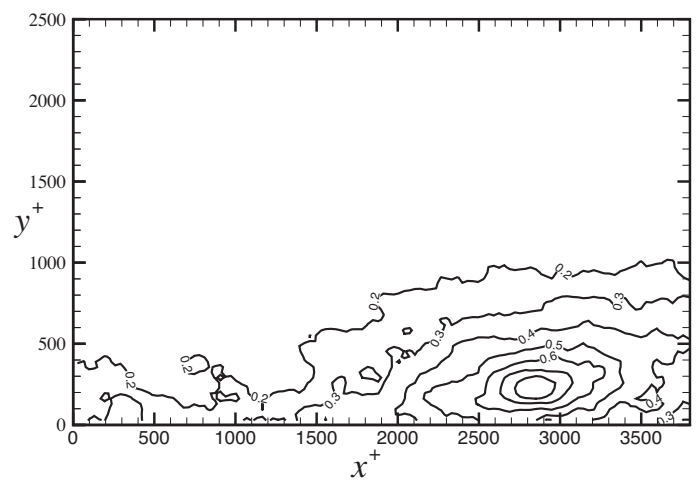

Figure 11: $\rho_{u u}\left(x^{+}, y^{+} ; x_{\mathrm{ref}}^{+}, y_{\mathrm{ref}}^{+}\right)$at streamwise location $M_{3}$ in plane 1 and different $\left(x_{\mathrm{ref}}^{+}, y_{\mathrm{ref}}^{+}\right)$ locations for hemisphere $\mathrm{A}$.
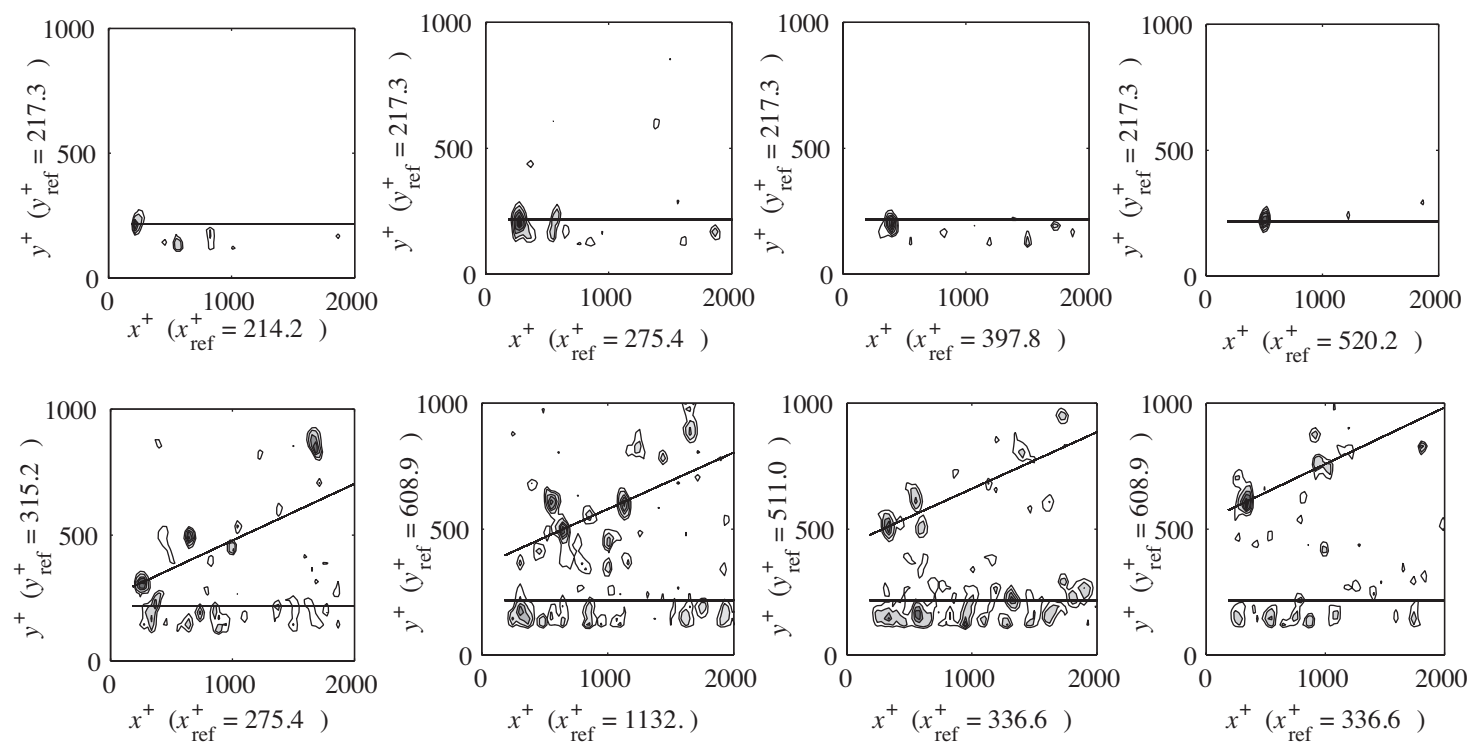

Figure 12: $\rho_{\lambda \lambda}\left(x^{+}, y^{+} ; x_{\text {ref }}^{+}, y_{\text {ref }}^{+}\right)$at different $\left(x_{\text {ref }}^{+}, y_{\text {ref }}^{+}\right)$locations for hemisphere B. 

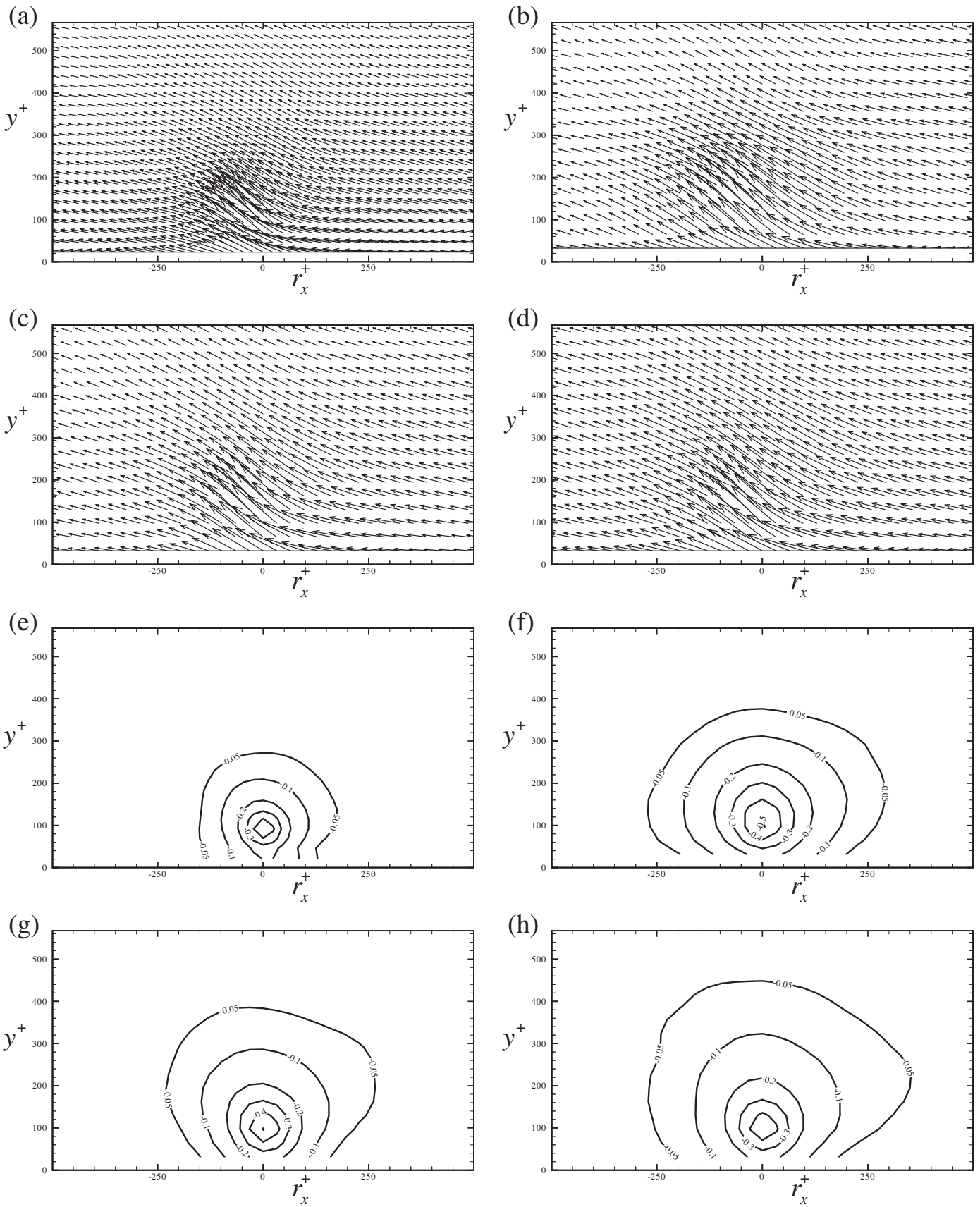

Figure 13: Linear stochastic estimates based on specification of a Q2 event at $y^{+} \simeq 100$. (a) Smooth wall, velocity vectors; (b) hemisphere A, plane 1, velocity vectors; (c) hemisphere A, plane 3, velocity vectors; (d) hemisphere A, plane 5, velocity vectors; (e) $u^{\prime} v^{\prime}$ contours for estimate, smooth wall; (f) $u^{\prime} v^{\prime}$ contours for estimate, hemisphere A, plane 1; (g) $u^{\prime} v^{\prime}$ contours for estimate, hemisphere A, plane 3 ; (h) $u^{\prime} v^{\prime}$ contours for estimate, hemisphere A, plane 5.

vortex that scales with $k$ rather than a perturbation in the buffer layer that scales with $\nu / u_{\tau}$. The original structure meanwhile is likely to feel the self-induced Q2 event and may i) reduce its convection velocity causing the new structures upstream to pack in the streamwise direction and ii) be lifted up from the wall by the enhanced $v$ fluctuation. 


\section{Conclusions}

We conclude that the presence of sparse roughness elements cause the shortening of the streamwise length scale in the near-wall region of the rough-wall flow under consideration. We confirm that vortex packets exist in the outer layer of flow over rough walls, but that their organization is altered, and we interpret this as the underlying cause of the length-scale reduction. The elements shed vortices, likely in an unsteady fashion (though the measurements could not capture this time-dependent phenomenon) which appear to align in the near-wall region, but are distinct from the packets. These shed vortices are a second population of structures that contribute to the correlation functions near the wall. Linear stochastic estimation with both homogeneous and inhomogeneous assumptions was required to extract the two populations from the correlation functions, and properly interpret the data. The interaction of these two populations results in the reduction of coherence in the near-wall region, through the alteration of the streamwise alignment of hairpins in the packet. This is due to i) the spanwise modulation of the region dominated by the element wakes and ii) the steepening of the packets in the outer layer. Both act to reduce the streamwise length scales both near the wall and in the outer layer. We do not have an exhaustive explanation on how exactly the vortices shed by the elements modify the inclination of the packets and thus alter the vortex organization in the outer layer. It is, however, inferred that the presence of the element as a rigid body extending into the log layer may act to deflect the packets upwards deeper into the outer layer. We also show that the $Q_{2}$ events triggered in the element wakes are more intense compared to the $Q_{2}$ events in smooth wall. We speculate that these two processes (deflection of the packet upward and triggering of particularly intense $Q_{2}$ events) may initiate the self-sustaining mechanism leading to the formation of hairpin packets as a much more effective instability compared to those typical of smooth-wall turbulence. Indeed the fluctuations of the wall-normal velocity associated with vortices of size comparable with the roughness elements, $k^{+}=100-200$, appear to be more intense as compared to those associated with the processes of tilting and bending of streamwise vorticity extending $20-50 y^{+}$, typical of the smooth-wall streaks.

\section{Notation}

$D_{z}=$ hemisphere spacing in spanwise direction $[\mathrm{m}]$

$\Delta U^{+}=$roughness function [-]

$\Delta x^{+}=$inner-scaled streamwise PIV grid spacing [-]

$\Delta y^{+}=$inner-scaled wall-normal PIV grid spacing [-]

$\Delta t=\mathrm{PIV}$ time delay $[\mu \mathrm{s}]$

$h=$ height of surface-mounted protuberance $[\mathrm{m}]$

$k=$ characteristic roughness height $[\mathrm{m}]$

$k_{s}=$ equivalent sand-grain roughness height $[\mathrm{m}]$

$l_{x}=$ streamwise field of view of PIV measurement $[\mathrm{m}]$

$l_{y}=$ wall-normal field of view of PIV measurement $[\mathrm{m}]$

$L_{x}=$ streamwise coherence of $\rho_{u u}[\mathrm{~m}]$

Re $=$ Reynolds number [-]

$r_{x}=$ spatial separation in the streamwise direction for two-point correlation calculation $[\mathrm{m}]$

$S=$ total surface area of roughness $\left[\mathrm{m}^{2}\right]$

$S=$ projected area of roughness $\left[\mathrm{m}^{2}\right]$

$\hat{u}=$ streamwise velocity at tip of surface-mounted protuberance $\left[\mathrm{m} \mathrm{s}^{-1}\right]$

$u_{\tau}=$ friction velocity $\left[\mathrm{m} \mathrm{s}^{-1}\right]$

$U_{c}=$ streamwise advection velocity for Galilean decomposition $\left[\mathrm{m} \mathrm{s}^{-1}\right]$ 
$U_{\infty}=$ free-stream velocity $\left[\mathrm{m} \mathrm{s}^{-1}\right]$

$u^{\prime}=$ streamwise velocity fluctuation $\left[\mathrm{m} \mathrm{s}^{-1}\right]$

$v^{\prime}=$ wall-normal velocity fluctuation $\left[\mathrm{m} \mathrm{s}^{-1}\right]$

$w^{\prime}=$ spanwise velocity fluctuation $\left[\mathrm{m} \mathrm{s}^{-1}\right]$

$x=$ streamwise direction $[\mathrm{m}]$

$y=$ wall-normal direction $[\mathrm{m}]$

$y_{*}=$ viscous length scale $[\mathrm{m}]$

$z=$ spanwise direction $[\mathrm{m}]$

$\delta=$ boundary-layer thickness $[\mathrm{m}]$

$\lambda=$ roughness density [-]

$\lambda_{c i}=$ swirling strength $\left[\mathrm{s}^{-1}\right]$

$\nu=$ kinematic viscosity $\left[\mathrm{m}^{2} \mathrm{~s}^{-1}\right]$

$\rho=$ density $\left[\mathrm{kg} \mathrm{m}^{-3}\right]$

$\rho_{u u}=$ two-point correlation coefficient of streamwise velocity [-]

$\rho_{\lambda \lambda}=$ two-point correlation coefficient of swirling strength [-]

$\sigma_{u}=$ root-mean-square of the streamwise velocity $\left[\mathrm{m} \mathrm{s}^{-1}\right]$

$\tau_{w}=$ wall shear stress $[\mathrm{Pa}]$

Subscripts

ref $=$ reference position for two-point correlation calculation

Superscripts

$+=$ normalization by $u_{\tau}$ and $\nu$

\section{References}

M. S. Acarlar and C. R. Smith. A study of hairpin vortices in a laminar boundary layer. J. Fluid Mech., 175(Part 1.):1-41, 1987a.

R. J. Adrian and I. Marusic. Coherent structures in flow over hydraulic surfaces. J. Hydraulic Res., in this issue, 2012.

R. J. Adrian and P. Moin. Stochastic estimation of organized turbulent structure: homogeneous shear flow. J. Fluid Mech., 190:531-559, 1988.

R. J. Adrian, K. T. Christensen, and Z. C. Liu. Analysis and interpretation of instantaneous turbulent velocity fields. Exp. Fluids, 29:275-290, 2000a.

R. J. Adrian, C. D. Meinhart, and C. D. Tomkins. Vortex organization in the outer region of the turbulent boundary layer. J. Fluid Mech., 422:1-54, 2000b.

J. J. Allen, M. A. Shockling, G. J. Kunkel, and A. J. Smits. Turbulent flow in smooth and rough pipes. Phil. Trans. R. Soc. A, 365:699-714, 2007.

O. M. Bakken, P. A. Frogstad, A. Ashrafian, and H. I Andersson. Reynolds number effects in the outer layer of the turbulent flow in a channel with rough walls. Phys. Fluids, 17(065101), 2005.

B. J. Balakumar and R. J. Adrian. Large- and very-large-scale motions in channel and boundarylayer flows. Phil. Trans. R. Soc. A, 365:665-681, 2007. 
P. R. Bandyopadhyay and R. D. Watson. Structure of rough-wall turbulent boundary layers. Phys. Fluids, 31:1877-1883, 1988.

G. L. Brown and A. S. W. Thomas. Large structure in a turbulent boundary layer. Phys. Fluids, 20:S243-S252, 1977.

I. P. Castro and A. G. Robins. The flow around a surface-mounted cube in uniform and turbulent streams. J. Fluid Mech., 79:307-335, 1977.

K. T. Christensen. The influence of peak-locking errors on turbulence statistics computed from piv ensembles. Experiments in Fluids, 36(3):484-497, 2004.

K. T. Christensen and R. J. Adrian. Statistical evidence of hairpin vortex packets in wall turbulence. J. Fluid Mech., 431:433-443, 2001.

K. T. Christensen and Y. Wu. Characteristics of vortex organization in the outer layer of wall turbulence. In 4th International Symposium on Turbulence and Shear Flow Phenomena, 2005.

K. T. Christensen, S. M. Soloff, and R. J. Adrian. Piv sleuth: Integrated particle-image velocimetry (PIV) interrogation/validation software. Technical Report 943, Department of Theoretical and Applied Mechanics, University of Illinois at Urbana-Champaign., 2000.

M. Detert, V. Nikora, and G. H. Jirka. Synoptic velocity and pressure fields at the water-sediment interface of streambeds. J. Fluid Mech., 660:55-86, 2010.

K. A. Flack, M. P. Schultz, and T. A. Shapiro. Experimental support for Townsend's Reynolds number similarity hypothesis on rough walls. Phys. Fluids, 17:035102, 2005.

O. Flores, J. Jimenez, and J. C. del Alamo. Vorticity organization in the outer layer of turbulent channels with disturbed walls. J. Fluid Mech., 591:145-154, 2007.

J. George. Structure of 2-D and 3-D Turbulent Boundary Layers with Sparsely Distributed Roughness Elements. PhD thesis, Virginia Polytechnic Institute and State University, 2005.

A. J. Grass. Structural features of turbulent flow over smooth and rough boundaries. J. Fluid Mech., 50:233-255, 1971.

J. Jimenez. Turbulent flow over rough wall. Annu. Rev. Fluid Mech., 36:173, 2004.

L. Keirsbulck, L. Labraga, A. Mazouz, and C. Tournier. Surface roughness effects on turbulent boundary layer structures. J. Fluids Eng., 124:127-135, 2002.

P. A. Krogstad and R. A. Antonia. Structure of turbulent boundary layers on smooth and rough walls. J. Fluid Mech., 277:1-21, 1994.

P. A. Krogstad and R. A. Antonia. Surface roughness effects in turbulent boundary layers. Exp. Fluids, 27:450-460, 1999.

P. A. Krogstad, R. A. Antonia, and L. W. B. Browne. Comparison between rough and smooth-wall turbulent boundary layers. J. Fluid Mech., 245:599-617, 1992.

G. J. Kunkel and I. Marusic. Study of the near-wall-turbulent region of the high-Reynolds-number boundary layer using an atmospheric flow. J. Fluid Mech., 548:375-402, 2006. 
S. H. Lee and H. J. Sung. Direct numerical simulation of the turbulent boundary layer over a rod-roughened wall. J. Fluid Mech., 584:125-146, 2007.

P. M. Ligrani and R. J. Moffat. Structure of transitionally rough and fully rough turbulent boundary layers. J. Fluid Mech., 162:69-98, 1986.

R. Martinuzzi and C. Tropea. The flow around surface-mounted, prismatic obstacles placed in a fully developed channel flow. J. Fluids Eng., 115:85-92, 1993.

C. D. Meinhart and R. J. Adrian. On the existence of uniform momentum zones in a turbulent boundary layer. Phys. Fluids, 7:694-696, 1995.

R. Mejia-Alvarez and K. T. Christensen. The impact of low-order representations of irregular surface roughness on flow in the roughness sublayer. AIAA Paper 2010-5018, 2010a.

R. Mejia-Alvarez and K. T. Christensen. Low-order representations of irregular surface roughness and their impact on a turbulent boundary layer. Phys. Fluids, 22:015106, 2010b.

R. Mejia-Alvarez, J. M. Barros, and K. T. Christensen. Structural attributes of turbulent flow over a complex topography. In M. Church J. Vendetti, J. Best and R. Hardy, editors, Coherent Flow Structures at the Earth's Surface. Wiley Blackwell, 2012.

S. Nakagawa and T. J. Hanratty. Particle image velocimetry measurements of flow over a wavy wall. Phys. Fluids, 13(11):3504-3507, 2001.

V. K. Natrajan, Y. Wu, and K. T. Christensen. Spatial signatures of retrograde spanwise vortices in wall turbulence. J. Fluid Mech., 574:155-167, 2007.

M. R. Raupach. Conditional statistics of reynolds stress in rough-wall and smooth-wall turbulent boundary layers. J. Fluid Mech., 108:363-382, 1981.

M. R. Raupach, R. A. Antonia, and S. Rajagopalan. Rough-wall turbulent boundary layers. Appl. Mech. Rev., 44:1-25, 1991.

A. G. Roy, T. B. Langer, H. Lamarre, and A. D. Kirkbride. Size, shape and dynamics of large-scale turbulent flow structures in a gravel-bed river. J. Fluid Mech., 500:1-27, 2004.

M. D. Ryan, C. Ortiz-Duenas, and E. K. Longmire. Effects of simple wall-mounted cylinder arrangements on a turbulent boundary layer. AIAA J., 49:2210-2220, 2011.

J. Sabot, I. Saleh, and G. Comte-Bellot. Effects of roughness on the intermittent maintenance of Reynolds shear stress in pipe flow. Phys. Fluids, 20, Pt II(10):S150-S155, 1977.

E. Savory and N. Toy. The flow regime in the turbulent near wake of a hemisphere. Exp. Fluids, 4 (4):181-188, 1986.

W. H. Schofield and E. Logan. Turbulent shear flow over surface mounted obstacles. J. Fluids Eng., 112:376-385, 1990.

M. F. Tachie, D. J. Bergstrom, and R. Balachandar. Rough wall turbulent boundary layers in shallow open channel flow. J. Fluids Eng., 122:533-541, 2000.

M. F. Tachie, D. J. Bergstrom, and R. Balachandar. Roughness effects in low-Re ${ }_{h}$ open-channel turbulent boundary layers. Exp. Fluids, 35:338-346, 2003. 
C. D. Tomkins. The structure of turbulence over smooth and rough walls. PhD thesis, University of Illinois at Urbana-Champaign, 2001.

C. D. Tomkins and R. J. Adrian. Spanwise structure and scale growth in turbulent boundary layers. J. Fluid Mech., 490:37-74, 2003.

R. J. Volino, M. P. Schultz, and K. A. Flack. Turbulence structure in rough- and smooth-wall boundary layers. J. Fluid Mech., 592:263-293, 2007.

R. J. Volino, M. P. Schultz, and K. A. Flack. Turbulence structure in a boundary layer with two-dimensional roughness. J. Fluid Mech., 635:75-101, 2009.

Y. Wu and K. T. Christensen. Population trends of spanwise vortices in wall turbulence. J. Fluid Mech., 568:55-76, 2006.

Y. Wu and K. T. Christensen. Outer-layer similarity in the presence of a practical rough-wall topography. Phys. Fluids, 19:085108, 2007.

Y. Wu and K. T. Christensen. Spatial structure of a turbulent boundary layer with irregular surface roughness. J. Fluid Mech., 655:380-418, 2010.

J. Zhou, R. J. Adrian, S. Balachandar, and T. M. Kendall. Mechanisms for generating coherent packets of hairpin vortices in channel flow. J. Fluid Mech., pages 353-396, 1999. 